candidates. Our patient had already recovered from septic shock and was no longer in a hypotensive and hyperdynamic circulatory state, which was probably a prerequisite for the high efficacy of ECMO support. Her prompt improvement and rapid recovery after ECMO insertion were remarkable and the course of ARDS in patients receiving ECMO support without invasive ventilation warrants further study. In patients with more severe lung injury one might also consider the use of ECMO in awake patients receiving noninvasive ventilation. To date, the use of ECMO in awake patients is investigational and must be carefully investigated before broader use.

Olaf Wiesner ${ }^{*,+}$, Johannes Hadem ${ }^{\#,+}$, Wiebke Sommer", Christian Kühn ", Tobias Welte* and Marius M. Hoeper* *Dept of Respiratory Medicine, Hannover Medical School, \#Dept of Gastroenterology, Hepatology and Endocrinology, Hannover Medical School, and "Dept of Cardiovascular, Thoracic and Transplantation Surgery, Hannover Medical School, Hannover, Germany. ${ }^{+}$These authors contributed equally to the manuscript.

Correspondence: M.M. Hoeper, Dept of Respiratory Medicine, Hannover Medical School, Carl-Neuberg-Str. 1, 30625 Hannover, Germany. E-mail: hoeper.marius@mh-hannover.de
Statement of Interest: None declared.

\section{REFERENCES}

1 Antonelli M, Conti G, Esquinas A, et al. A multiple-center survey on the use in clinical practice of noninvasive ventilation as a first-line intervention for acute respiratory distress syndrome. Crit Care Med 2007; 35: 18-25.

2 Peek GJ, Mugford M, Tiruvoipati R, et al. Efficacy and economic assessment of conventional ventilatory support versus extracorporeal membrane oxygenation for severe adult respiratory failure (CESAR): a multicentre randomised controlled trial. Lancet 2009; 374: 1351-1363.

3 Fuehner T, Kuehn C, Hadem J, et al. Extracorporeal membrane oxygenation in awake patients as bridge to lung transplantation. Am J Respir Crit Care Med 2012; 185: 763-768.

4 Olsson KM, Simon A, Strueber M, et al. Extracorporeal membrane oxygenation in nonintubated patients as bridge to lung transplantation. Am J Transplant 2010; 10: 2173-2178.

5 Crotti S, Lissoni A, Tubiolo D, et al. Artificial lung as an alternative to mechanical ventilation in COPD exacerbation. Eur Respir J 2012; 39: 212-215.

6 Murray JF, Matthay MA, Luce JM, et al. An expanded definition of the adult respiratory distress syndrome. Am Rev Respir Dis 1988; 138: 720-723.

DOI: $10.1183 / 09031936.00076912$

\title{
Pleural effusion arising from a rare pancreatic neoplasm
}

\section{To the Editors:}

Pleural effusions are common entities and may complicate a number of disease processes. We present the case of a large pleural effusion associated with a rare pancreatic neoplasm. The patient, a 67-yr-old female, was referred for respiratory opinion by the Breast Cancer Service at St Vincent's University Hospital (Dublin, Ireland). She had a background of invasive ductal carcinoma of the right breast 4 yrs previously for which she had undergone a wide local excision and was taking hormonal therapy. Other past medical history included a diagnosis of seropositive rheumatoid arthritis requiring only analgesic therapy. She had known tuberculosis (TB) exposure in childhood and was a nonsmoker. She drank alcohol only on occasion.

She had initially noticed that she was sinking to the left side while swimming over the previous month. This was followed by progressive dyspnoea on exertion, left-sided chest pain and nocturnal non-productive cough. She denied haemoptysis or weight loss and was systemically well. Physical examination identified stony-dull percussion and reduced breath sounds over the mid-lower left lung. She was comfortable at rest with oxygen saturations of $96 \%$ on room air. There was no clubbing or lymphadenopathy. A chest radiograph confirmed a large leftsided pleural effusion (fig. 1a). Pleural fluid analysis was consistent with an exudative effusion with elevated fluid protein and lactate dehydrogenase $\left(39 \mathrm{~g} \cdot \mathrm{L}^{-1}\right.$ and $1,126 \mathrm{~g} \cdot \mathrm{L}^{-1}$, respectively). Cytology was negative for malignant cells and microbiology testing failed to identify any organisms, including acid-fast bacilli. Immunohistochemistry staining for thyroid transcription factor-1 and oestrogen receptor were negative.

Full blood count and biochemical markers were all within normal limits. Serum tumour markers were negative. Rheumatoid factor was positive with negative antinuclear antibody and antineutrophilic cytoplasmic antibody. The Mantoux test was negative.

Computed tomography of the thorax, abdomen and pelvis performed prior to respiratory referral confirmed a large leftsided effusion with almost complete collapse of the left lung and no obvious endobronchial lesion. A $3 \times 3.6 \mathrm{~cm}$ left-sided juxta-renal fluid collection was identified in the upper abdomen (fig. 1b). This had been identified 4 yrs previously on abdominal ultrasound and was unchanged in size. The remaining abdominal examination appeared normal.

Initial ultrasound-guided thoracocentesis yielded over $1.5 \mathrm{~L}$ of blood-stained fluid. A wide-bore chest drain was inserted when the fluid rapidly re-accumulated causing worsening dyspnoea.

Subsequent video-assisted thorascopic surgery removed a further 1.3 L of blood-stained fluid. Pleural biopsy revealed reactive 

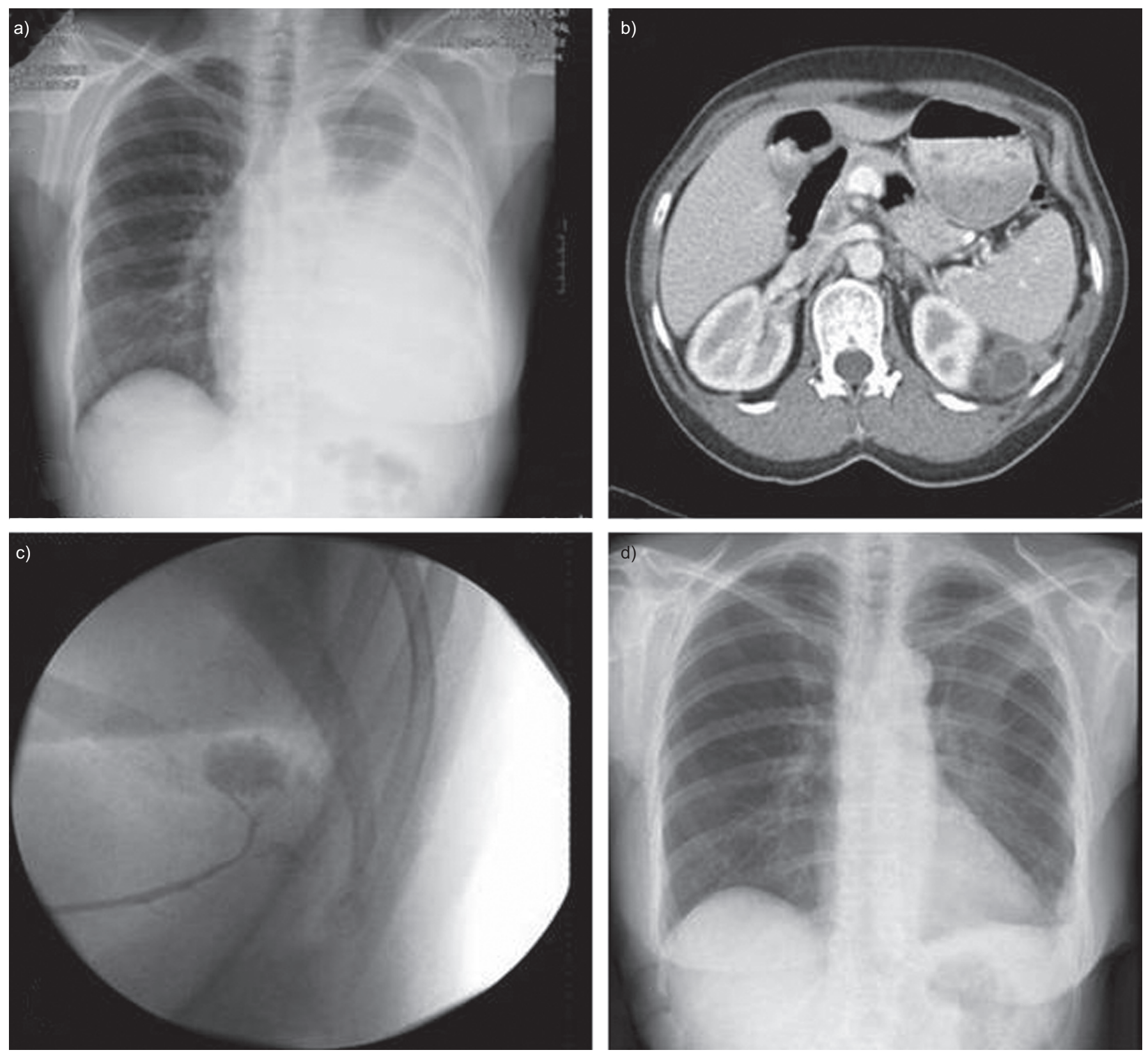

FIGURE 1. a) Chest radiograph on admission with large left-sided pleural effusion. b) Computed tomography of the abdomen revealing left-sided subdiaphragmatic collection. c) Endoscopic retrograde cholangiopancreatogram showing leakage of contrast from the pancreatic tail. d) Repeat chest radiograph 7 days post-surgery with almost complete resolution of the pleural effusion.

inflamed pleural tissue with evidence of fibrosis. Pleural fluid amylase was measured and returned at $1,716,000 \mathrm{U} \cdot \mathrm{L}^{-1}$ (serum amylase of $\left.220 \mathrm{U} \cdot \mathrm{L}^{-1}\right)$.

The extremely elevated fluid amylase in this rapidly recurring pleural effusion of no obvious aetiology, and in the presence of a long-standing upper abdominal collection, raised the suspicion of a possible pancreaticopleural fistula.

Endoscopic retrograde cholangiopancreatogram (ERCP) revealed leakage of contrast from the pancreatic tail into the previously identified juxta-renal fluid collection (fig. 1c). An explorative laporotomy found a cystic collection on the posterior pancreatic surface and distal pancreatectomy was performed. Histology confirmed an intraductal papillary mucinous neoplasm (IPMN) of the pancreas with evidence of low-grade dysplasia. An inflamed tract was visualised at the distal pancreatic margin. The patient made an uncomplicated post-operative recovery, the pleural effusion resolved and she was discharged 1 week later (fig. 1d).

In summary, this patient had a rapidly recurring and persistent pleural effusion with a number of obvious risk factors for developing a pleural effusion, namely malignancy, rheumatoid arthritis and previous TB exposure. It was ultimately the finding of the markedly elevated pleural fluid amylase, after initial routine fluid analysis failed to identify a possible cause 
for the effusion, which led to the diagnosis of pancreaticopleural fistula with histology confirming that it arose from a pancreatic intraductal papillary mucinous neoplasm.

While pleural effusions are common, those secondary to pancreaticopleural fistulae are rare, most often occurring in patients with a history of chronic pancreatitis or alcoholism [1]. In this case the fistula formed from an IPMN. These are extremely rare entities that represent $10 \%$ of pancreatic cysts. They can show varying degrees of dysplasia with recurrence being rare in noninvasive types $(<8 \%)$ versus invasive types (50-65\%) [2]. Fistulae to abdominal viscera have been described previously [3]; however, to our knowledge, they have not been known to fistulate to the pleural cavity.

Management options are not well defined due to the paucity of cases, but include therapeutic thoracocentesis in conjunction with total parenteral nutrition or somatostatin therapy in an attempt to reduce pancreatic secretions. Interventional approaches involve ERCP with pancreatic duct stenting, pseudocyst drainage or distal pancreatectomy.

Measurement of pleural fluid amylase raised the possibility of a pancreaticopleural fistula in this case. Pleural fluid amylase may be elevated in a number of cases including pancreatic disease, oesophageal rupture or malignancy. A strong association has been shown between amylase-rich effusions and malignancy, most commonly primary lung carcinoma [4]; however, there remains considerable debate over the benefit of measuring pleural fluid amylase in clinical practice. In 2001, BRANCA et al. [5] measured amylase levels in 379 pleural effusions and found that only $1.3 \%$ of cases had an amylase level of $>100 \mathrm{U} \cdot \mathrm{L}^{-1}$. In no case did amylase measurement assist in determining the origin of the effusion [5]. While an association has been shown between amylase-rich effusions and malignancy, only $10-15 \%$ of the malignant effusions in that study were rich in amylase [4]. Currently, the British Thoracic Society guidelines do not recommend routine measurement of pleural fluid amylase [6].

While we do not advocate the routine testing of pleural fluid amylase, this case demonstrates that it may be a worthwhile consideration in cases where the aetiology of an effusion remains unclear.

Our patient remains well and at most recent follow-up, $>18$ months post-surgery, the effusion has not recurred.

\section{Breda Cushen*, Aoife McKeating*, John F. Garvey*,} Jonathan D. Dodd", Hugh Mulcahy ${ }^{\oplus}$, Justin Geoghegan ${ }^{+}$, Edward F. McKone* and Charles G. Gallagher* *Dept of Respiratory Medicine, St. Vincent's University Hospital, "Dept of Radiology, St. Vincent's University Hospital, 'Dept of Gastroenterology, St. Vincent's University Hospital, and ${ }^{+}$Dept of Hepatobiliary Surgery, St. Vincent's University Hospital, Dublin, Ireland.

Correspondence: C.G. Gallagher, National Referral Centre for Adult Cystic Fibrosis, St. Vincent's University Hospital, Elm Park, Dublin 4, Ireland. E-mail: c.gallagher@st-vincents.ie

Statement of Interest: None declared.

\section{REFERENCES}

1 Rockey DC, Cello JP. Pancreaticopleural fistula. Report of 7 patients and review of the literature. Medicine (Baltimore) 1990; 69: 332-344.

2 Bassi C, Sarr MG, Lillemoe KD, et al. Natural history of intraductal papillary mucinous neoplasms (IPMN): current evidence and implications for management. J Gastrointest Surg 2008; 12: 645-650.

3 Shimizu M, Kawaguchi A, Nagao S, et al. A case of intraductal papillary mucinous neoplasm of the pancreas rupturing both the stomach and duodenum. Gastrointest Endosc 2010; 71: 406-412.

4 Villena V, Pérez V, Pozo F, et al. Amylase levels in pleural effusions: a consecutive unselected series of 841 patients. Chest 2002; 121: 470-474.

5 Branca P, Rodriguez RM, Rogers JT, et al. Routine measurement of pleural fluid amylase is not indicated. Arch Intern Med 2001; 161: 228-232.

6 Hooper C, Lee YC, Maskell N, et al. Investigation of a unilateral pleural effusion in adults: British Thoracic Society Pleural Disease Guideline 2010. Thorax 2010; 65: Suppl. 2, ii4-17.

DOI: $10.1183 / 09031936.00035312$

\section{Lung toxicity in a patient treated with sunitinib}

\section{To the Editors:}

A 61-yr-old male presented in 2008 with dyspnoea on exertion and night sweats. Diagnosis of mixed connective tissue disease with pulmonary fibrosis was made. The Latex test, Waaler-Rose test and antinuclear antibodies (anti-centromeres) were positive. The computed tomography (CT) image of the abdomen and pelvis was considered normal at that time. He was started on steroids and the dyspnoea improved. Respiratory functional tests remained abnormal with decreased diffusion capacity of the lung for carbon monoxide (DL,CO) (50\%). 6 months later, in April 2009, he developed a fever and macroscopic haematuria. The CT image showed a $9 \mathrm{~cm}$ tumour in the left kidney with latero-aortic lymph nodes and multiple lung metastases (fig. 1a).
There was also evidence of lung fibrosis. A biopsy of the kidney tumour and a lymph node was performed. The results showed evidence of tubulopapillary renal carcinoma (Fürhrman grade II) in the kidney biopsy. The lymph node was involved by a poorly differentiated nonsmall cell carcinoma, different from the kidney lesion. He was referred to the cancer centre. The bone scan and brain magnetic resonance imaging were normal. A left radical nephrectomy with retroperitoneal lymph-node dissection was performed. Pathological examination revealed a $12 \mathrm{~cm}$ high-grade (Führman grade IV) mixed renal-cell carcinoma (clear cell carcinoma, tubulopapillary carcinoma and a sarcomatoid component) with lymph node involvement. It was a pT3a pN2 M1 tumour according to the tumour, node, metastasis 